OPEN ACCESS

Edited by:

Kristijan Ramadan,

University of Oxford, UK

Reviewed by:

Richard Chahwan,

Albert Einstein College of Medicine,

Andrew N. Blackford,

University of Cambridge, UK

*Correspondence:

Tony T. Huang

tony.huang@nyumc.org

Specialty section:

This article was submitted to

Cancer Genetics,

a section of the journal

Frontiers in Genetics

Received: 03 March 2016 Accepted: 01 April 2016

Published: 19 April 2016

Citation:

Coleman KE and Huang TT (2016)

How SUMOylation Fine-Tunes

the Fanconi Anemia DNA Repair

Pathway. Front. Genet. 7:61.

doi: 10.3389/fgene.2016.00061

\section{How SUMOylation Fine-Tunes the Fanconi Anemia DNA Repair Pathway}

\author{
Kate E. Coleman and Tony T. Huang* \\ Department of Biochemistry and Molecular Pharmacology, New York University School of Medicine, New York, NY, USA
}

Fanconi anemia (FA) is a rare human genetic disorder characterized by developmental defects, bone marrow failure and cancer predisposition, primarily due to a deficiency in the repair of DNA interstrand crosslinks (ICLS). ICL repair through the FA DNA repair pathway is a complicated multi-step process, involving at least 19 FANC proteins and coordination of multiple DNA repair activities, including homologous recombination, nucleotide excision repair and translesion synthesis (TLS). SUMOylation is a critical regulator of several DNA repair pathways, however, the role of this modification in controlling the FA pathway is poorly understood. Here, we summarize recent advances in the fine-tuning of the FA pathway by small ubiquitin-like modifier (SUMO)targeted ubiquitin ligases (STUbLs) and other SUMO-related interactions, and discuss the implications of these findings in the design of novel therapeutics for alleviating FA-associated condition, including cancer.

\begin{abstract}
Keywords: Fanconi anemia (FA), SUMOylation, SUMO-targeted ubiquitin ligases (STUbLs), SUMO proteases (SENPs), SUMO-interaction motifs (SIMs), SUMO-like domains (SLDs), deubiquitinases (DUBs), DNA interstrand crosslinks (ICLs)
\end{abstract}

\section{FANCONI ANEMIA (FA) DNA REPAIR PATHWAY}

Fanconi anemia is a very rare autosomal recessive disease (occurring in just 1 of every 100,000 births), typically distinguished by bone marrow abnormalities, cancer predisposition, and congenital anomalies (Kee and D'Andrea, 2012). Patients with this disease exhibit defects in the repair of interstrand crosslinks (ICLs), DNA lesions which hook bases of opposing DNA strands together, and are consequently hypersensitive to crosslinking agents such as cisplatin, diepoxybutane (DEB), and mitomycin C (MMC) (Noll et al., 2006). Despite the low prevalence of the FA disease, the complex pathway involved in the recognition and repair of ICLs orchestrates multiple DNA repair processes, such as homologous recombination (HR) and translesion synthesis (TLS), making FA an important model in the study of DNA repair signaling.

To date, 19 FANC proteins have been identified in the FA pathway, which fall into three distinct functional groups. One group is the FA core complex, consisting of FANCA, B, C, E, F, G, L, and M, which together with three Fanconi associated proteins (FAAP20, FAAP24, and FAAP100) functions as an E3 ubiquitin ligase (Garcia-Higuera et al., 2001; Meetei et al., 2003; Ciccia et al., 2007; Ling et al., 2007; Leung et al., 2012). Upon pathway induction by DNA damage or replication stress, the targeting components of the FA core, FANCM and FAAP24, bind chromatin and recruit the FA core complex (Qiao et al., 2001; Kim et al., 2008). A histone-fold-containing complex consisting of MHF1-MHF2 proteins facilitates the recruitment of FANCM to chromatin to enhance 
pathway activation (Singh et al., 2010; Yan et al., 2010). The FA core complex subsequently monoubiquitylates group II proteins, FANCI and FANCD2, which associate to form a heterodimer called the ID complex (Garcia-Higuera et al., 2001; Sims et al., 2007; Smogorzewska et al., 2007). This monoubiquitylation of the ID complex localizes it to chromatin, where it recruits the group III effector proteins to initiate downstream ICL repair.

Following ID monoubiquitylation, a complex series of steps ensues to complete ICL repair. First, the mono-ubiquitinated ID complex serves as a scaffold for recruitment of several nucleases, which make nucleolytic incisions flanking the ICL to unhook the crosslink. These nucleases include SLX4 (discussed in more detail below), ERCC1-XPF1, and MUS81-EME1 structure-specific endonucleases (Hanada et al., 2006; Knipscheer et al., 2009; Stoepker et al., 2011; Klein Douwel et al., 2014). Cross-linked nucleotides on the complementary strand are subsequently bypassed by the process of TLS, which involves specialized Y-family polymerases such as pol $\zeta$ and REV1 (Räschle et al., 2008; Kim et al., 2012; Budzowska et al., 2015). The nucleolytic incisions result in a double strand break (DSB) in the DNA, which is repaired by HR (discussed below), and nucleotide excision repair (NER) fills the remaining gap (Räschle et al., 2008; Kim and D'Andrea, 2012). Finally, in the last step, the deubiquitinating (DUB) complex USP1-UAF1 removes monoubiquitin from the ID complex, allowing for pathway regeneration (Nijman et al., 2005; Cohn et al., 2007) (Figure 1). For more extensive reviews on the steps of the FA pathway, please refer to the following reviews: (Kim and D'Andrea, 2012; Walden and Deans, 2014; Duxin and Walter, 2015). Despite extensive study, it is likely that additional aspects of FA pathway activation and regulation remain unidentified, prompting recent studies of other regulatory proteins and post-translational modifications (PTMs) involved in the FA pathway.

\section{CONTROL OF SIGNALING PATHWAYS BY SUMOylation}

In addition to ubiquitin signaling, modification of proteins by small ubiquitin-like modifier (SUMO) has been implicated in several aspects of cellular signaling. Similar to protein ubiquitylation, the process of activating and conjugating SUMO modifications to substrates involves an E1-E2-E3 enzyme cascade. SUMO proteins are initially translated as immature precursors that must be processed by proteases to a mature form containing a C-terminal diglycine motif. This mature form of SUMO subsequently becomes bound by an E1 enzyme that activates SUMO through sequential adenylation and thioester bond formation. SUMO is then passed to a single E2 conjugating enzyme, ubiquitin-like conjugating enzyme 9 (Ubc9), that transfers the SUMO to substrate accepter lysine residues with the help of E3 protein ligases. Protein SUMOylation is mediated by several SUMO E3 ligases in mammalian cells, including those of the PIAS family: PIAS1, PIAS2 (PIASx $\alpha / \beta)$, PIAS3, and PIAS4 (PIASy). These E3 enzymes facilitate SUMO conjugation either by promoting specificity to substrate recruitment by E2 SUMO, or by stimulating the discharge of SUMO to substrates (Gareau and Lima, 2010). The E2 enzyme Ubc9 recognizes its substrates through the consensus SUMOylation motif, $\Psi \mathrm{KxE} / \mathrm{D}$, where $\Psi$ is a large hydrophobic residue (Rodriguez et al., 2001). Conversely, SUMO can also be deconjugated from substrates through the activity of SUMO specific proteases (SENPs), making this a highly dynamic modification analogous to ubiquitylation and phosphorylation (Kumar and Zhang, 2015).

Another family of proteins called SUMO-targeted ubiquitin ligases (STUbLs) has also been characterized recently that connects the processes of SUMOylation with ubiquitylation. Uniquely, STUbL enzymes recognize their substrates through SUMO-interaction motifs (SIMs), short hydrophobic peptide sequences that mediate non-covalent attachments with SUMO (Perry et al., 2008). Often, STUbL enzymes promote the specific recruitment of proteins containing both ubiquitin-interacting motifs (UIMs) and SIMs through the synthesis of SUMOubiquitin conjugate chains. As an example, the RAP80 subunit of the BRCA1 complex uses both its SIM and UIM motifs to bind SUMO-ubiquitin conjugate chains synthesized by the STUbL RNF4 for efficient recruitment to DSBs immediately following DNA damage (Guzzo et al., 2012; Hu et al., 2012). By promoting the degradation of previously SUMOylated target proteins, STUbL enzymes play important roles as global regulators of SUMOylation levels. Imbalances in global protein SUMOylation levels can have several adverse cellular consequences, including genome instability and sensitivity to genotoxic stress (Perry et al., 2008).

Small ubiquitin-like modifier and SUMO-like modifications can influence a wide variety of cellular signaling pathways by directing changes in protein-protein interactions, altering protein intracellular localization, directing protein turnover (via STUbL enzymes described above), or changing protein activity. Despite its importance in these other signaling pathways (reviewed in Gareau and Lima, 2010), a functional role for ubiquitinSUMO crosstalk in the Fanconi Anemia pathway has not been defined until recently. This review highlights new evidence of FA pathway modulation by SUMO modifications and SUMOlike interactions, and describes the impact of these observations on our understanding of FA pathway regulation and disease treatment.

\section{ROLE OF FANCA SUMOylation}

One example of how SUMOylation contributes to FA pathway regulation is through promoting the polyubiquitylation of FA core complex member FANCA. A recent study from the D'Andrea lab identified a patient with a point mutation in FANCA (FANCA ${ }^{\mathrm{I935S}}$ ) that fails to bind the FAAP20 subunit of the FA core complex, leading to decreased FANCA protein levels. In uncovering the mechanism behind this decreased stability of FANCA, the authors discovered that defective FAAP20 binding by the FANCA mutant leads to increased exposure of a SUMOylation site on FANCA at residue K921, which in turn promotes UBC9-mediated SUMOylation, polyubiquitylation by the STUbL RNF4, and proteasome-dependent degradation of 


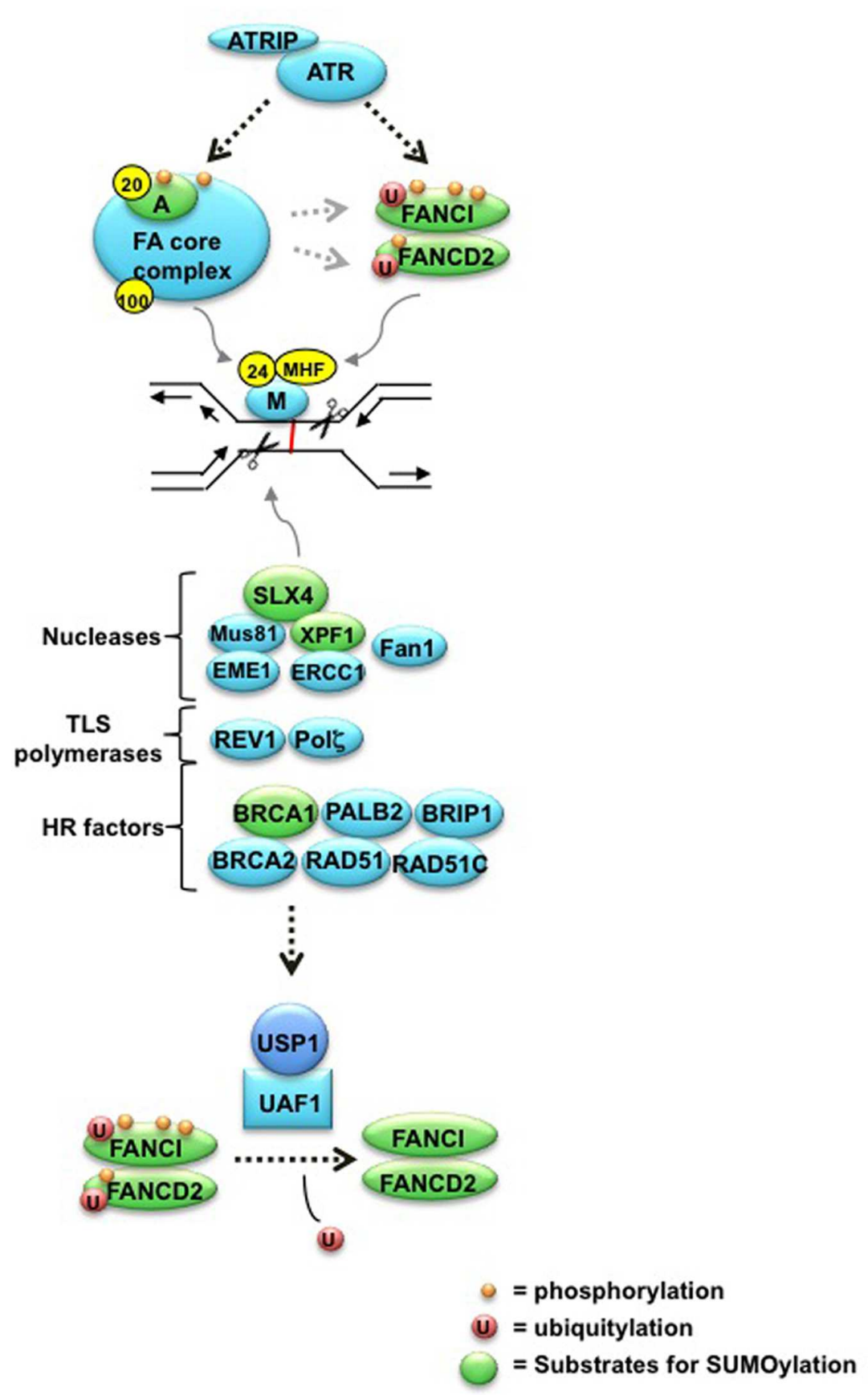

FIGURE 1 | The ICL repair pathway. The FANCM-FAAP24 complex first recognizes stalled replication structures, and recruits the FA core complex to the ICL. ATR-Chk1 phosphorylates and activates multiple components, including FANCA, E, I, and D2, which is required for monoubiquitylation of the ID complex by the FA core complex. Upon monoubiquitylation, the activated ID complex localizes to chromatin and coordinates the activities of downstream nucleases that create incisions around the crosslink. The resulting structure is then repaired by multiple processes, involving lesion bypass by TLS polymerases, HR, and NER. Finally, the USP1-UAF1 DUB complex removes monoubiquitin from the ID complex to complete repair. See accompanying text for more details. Yellow spheres indicate Fanconi Anemia-associated proteins (FAAPs) FAAP20, FAAP24, and FAAP100. Targets for SUMOylation highlighted in this review are denoted in green.

FANCA. Failure of the FANCA mutant to bind FAAP20 still allows efficient FANCD2 monoubiquitylation, but leads to an inability to properly recruit the REV1 translesion polymerase (Kim et al., 2012), contributing to lower rates of
TLS-mediated mutagenesis. On the other hand, the authors showed that wild-type FANCA is also subject to SUMOylation and RNF4-mediated polyubiquitylation, but to a lesser degree, and depletion of RNF4 contributed to increased MMC sensitivity 
(Xie et al., 2015) (Figure 2A). Taken together, these results indicate that the regulated release of FAAP20 from FANCA is a normal critical step in the FA pathway and suggest that failure to properly release FANCA from the FA core complex could contribute to pathway disruption and genome instability.

\section{SUMOylation OF THE ID COMPLEX FOR THE FA PATHWAY}

As discussed previously, ID complex monoubiquitylation is removed by the USP1-UAF1 (WDR48) DUB complex, allowing for FA pathway regeneration (Nijman et al., 2005; Cohn et al., 2007). Evidence shows that depletion of USP1 in either murine models or chicken DT40 cells enhances chromatin loading of the ID complex in the absence of exogenous DNA damage (Oestergaard et al., 2007; Kim et al., 2009), but only to similar levels as observed following mitomycin C (MMC) treatment, suggesting the existence of alternative mechanisms to restrain ID complex loading at DNA lesions. Work by GibbsSeymour et al. (2015) demonstrates that direct SUMOylation of the ID complex can also stimulate the removal of activated ID complexes at sites of damage, thereby controlling ID complex dosage at DNA lesions. ID complex SUMOylation is dependent on the ATR kinase and two SUMO E3 ligases, PIAS1 and PIAS4, and is antagonized by the SUMO protease SENP6. Following SUMOylation, the STUbL RNF4 targets the ID complex for polyubiquitylation, ultimately promoting ID complex chromatin extraction from DNA lesions by the DVC1p97 ubiquitin segregase complex. This ubiquitin-SUMO network thereby helps fine-tune ID complex recruitment to DNA damage sites. The authors further show that dysregulation of this process through expression of a SUMOylation-deficient FANCI mutant results in increased DNA damage and MMC sensitivity, illustrating the importance of this mechanism for limiting ID complex dosage at DNA lesions (Gibbs-Seymour et al., 2015) (Figure 2B).

An obvious follow-up question to this study is: why is it so important to precisely regulate ID complex dosage at DNA damage sites by this elaborate mechanism? The authors suggest that this regulation by ubiquitin-SUMO crosstalk could help prevent excessive nucleolytic processing of DNA, downstream of ID complex monoubiquitylation. Like USP1mediated deubiquination, SUMOylation of ID proteins could also be an important mechanism allowing for ID complex recycling and FA pathway regeneration. Future work will be needed to further understand mechanistically how dysregulation of ID complex dosage at DNA damage sites contributes to genome instability.

\section{SLX4 ACTS AS A SUMO E3 LIGASE}

One of the key steps in ICL repair is nucleolytic excision of the cross-link and downstream repair of the resulting DSB by HR. A key player that coordinates these complex repair processes is the SLX4 protein. Together with its activating subunit, SLX1, SLX4 associates with XPF-ERCC1 and MUS81EME1 structure-specific endonucleases to cleave branched DNA structures (Andersen et al., 2009; Munoz et al., 2009; Stoepker et al., 2011). SLX4 additionally interacts with the mismatch repair proteins MSH2-MSH3 and is required for telomere stability through association with TRF2 (Wan et al., 2013). SLX1SLX4 complexes serve as HJ resolvases and process multiple recombination intermediates (Fekairi et al., 2009). Thus, SLX4 serves as a scaffold for many different nucleases involved in ICL and HR repair, but our understanding of how these interactions are orchestrated to direct specific repair outcomes has been limited.

New studies from the Gaillard, Vertegaal, and Zou labs have shed light on SLX4 regulation, showing that SUMO and ubiquitin modifications are involved in directing the different activities of the SLX4 complex. Guervilly et al. (2015) made the surprising discovery that the SLX4 complex is a SUMO E3 ligase that SUMOylates SLX4 itself. SUMOylation by SLX4 is dependent on an interaction with the charged UBC9 SUMO E2 enzyme as well as newly identified SIMs in SLX4. Studies from the Vertegaal lab identified three such SIMs in the SLX4 protein and showed that these motifs were critical for proper ICL repair and targeting of SLX4 to PML nuclear bodies and laser-induced DNA damage sites (González-Prieto et al., 2015). In addition to SUMOylating itself, SLX4 also targets the XPF subunit of the repair endonuclease XPF-ERCC1 for SUMO modification (Guervilly et al., 2015). Unexpectedly, the BTB domain of SLX4, which facilitates XPF targeting (Andersen et al., 2009), was specifically required for SUMOylation of XPF in vivo and in vitro, showing an additional role for the BTB domain outside of XPF binding (Figure 2C). At one extreme, chronic overexpression of SLX4 induces global replication stress and is extremely cytotoxic, which the authors suggest are consequences of extensive nucleolytic processing and chromatid breakage. On the other hand, the SUMO E3 ligase activity of SLX4 facilitates expression of common fragile sites (CFS), unstable genomic loci that are difficult to replicate. In fact, failure to localize SLX4 to CFS is associated with increased anaphase bridges and mitotic catastrophe. Therefore, on a global scale, increased SUMO ligase activity of SLX4 is detrimental as it contributes to replication stress, but is necessary to prevent mitotic catastrophe following CFS expression (Guervilly et al., 2015).

Ouyang et al. (2015) made similar findings about the involvement of SUMOylation in SLX4 activity. Namely, they also discovered that SLX4 binds SUMO2/3 chains via SIMs in a manner dependent on charged UBC9, and that SUMO interactions are important for suppressing fragile site instability and processing of CPT-induced replication intermediates. However, the authors extend these findings by directly comparing SLX4 targeting via its ubiquitin-binding zinc finger (UBZ) domains versus its SIM domains. While the UBZs of SLX4 are critical in ICL repair, the SIMs of SLX4 are instead more important for binding DNA damage sensors such as RPA, the MRN complex, and the telomere binding protein TRF2. Thus, the UBZs and SIMs 


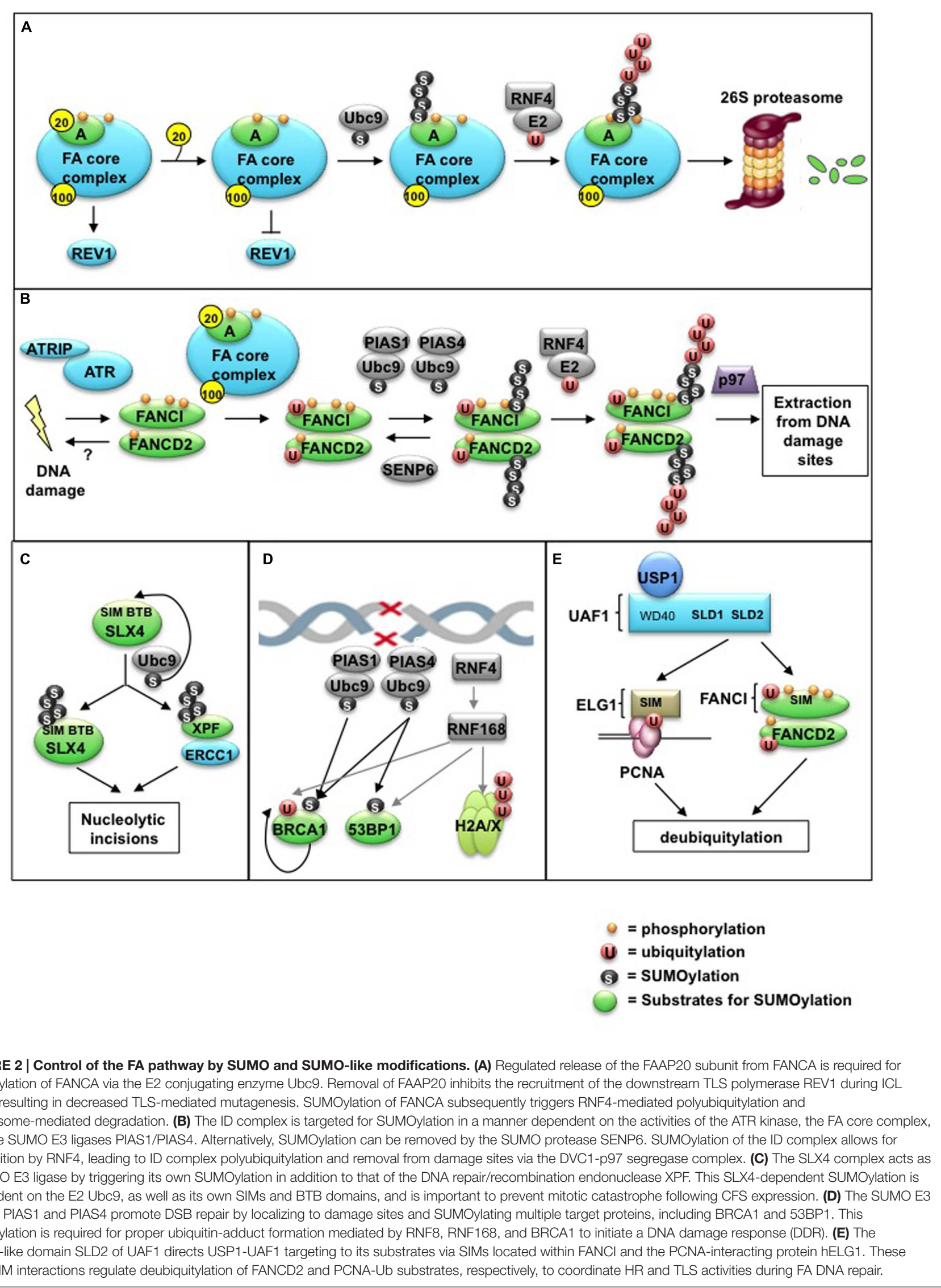


of SLX4 are functionally distinct, providing a mechanism for targeting SLX4 in different repair contexts (Ouyang et al., 2015).

\section{ROLE OF SUMOylated PCNA IN MAMMALIAN CELLS}

The FA pathway is important for activation of HR-mediated repair, a DSB repair mechanism that uses an intact sister chromatid as a repair template (review in Moynahan and Jasin, 2010). While HR has the advantage of being an errorfree mode of repair as opposed to the non-homologous end joining (NHEJ) pathway, inappropriate hyperrecombination is associated with genomic instability and cancer (Martin et al., 2007). How the FA pathway coordinates crosslink repair and HR appropriately has been a poorly understood topic. It is known that in budding yeast, the UvrD domain helicase Srs2 limits inappropriate HR by removing RAD51 nucleofilaments from ssDNA at an early step in the HR pathway (Papouli et al., 2005). A homolog for Srs2 with similar antirecombinase activity had not been identified in human cells, however, prompting a recent study by the D'Andrea lab to identify novel HR regulators in mammalian cells. The authors identified C12orf48 as an anti-recombinase in human and chicken DT40 cells that specifically interacts with a PCNA-SUMO fusion protein in vitro. They named this protein PCNA associated recombination inhibitor (PARI). Similar to Srs2 in yeast, PARI restricts recombination by interfering with RAD51-DNA HR structures and is required for genome stability. Interestingly, PARI knockdown in FA cells, which are deficient in HR (Nakanishi et al., 2005), improves genome instability by increasing recombination frequency (Moldovan et al., 2012). These results indicate that manipulation of PARI levels could be an effective chemoprotective approach in HR-deficient cancers. Nevertheless, several key questions still remain pertaining to the role and recruitment of PARI to the replication fork, including whether SUMOylation of mammalian PCNA exists and whether PARI is truly the long sought-after functional human homolog of budding yeast Srs2.

\section{ROLE OF SUMOylation IN HR REPAIR}

Following unhooking of an ICL by nucleolytic incisions, a DSB is generated that must be repaired by HR. Current evidence demonstrates a role for SUMOylation in coordinating this DSB repair step. SUMO E3 ligases PIAS1 and PIAS4 accumulate at DNA damage sites in mammalian cells, where they target multiple substrates for SUMO modification, including 53BP1 and BRCA1, to damage foci. SUMOylation facilitates the localization of these DNA repair proteins to DSB sites and also is required for RNF8 and RNF168-mediated ubiquitylation of target proteins (including $\mathrm{H} 2 \mathrm{~A} / \mathrm{X}$ ) to signal downstream repair (Galanty et al., 2009). In particular, SUMOylation of BRCA1 by PIAS SUMO E3 ligases increases its ubiquitin ligase activity, identifying it as a STUbL (Morris et al., 2009). In addition to
BRCA1, another STUbL, RNF4, was identified that promotes DSB repair by regulating the turnover of DSB-responsive factors $\mathrm{MDC} 1$ and replication protein A (RPA), allowing for recruitment of factors necessary for DSB repair by HR (Galanty et al., 2012). Through the combination of these mechanisms (Figure 2D), SUMO-ubiquitin crosstalk amplifies DSB signaling to promote efficient DSB repair. Although likely, it is currently unclear whether regulation of BRCA1 by SUMOylation plays a role in the repair of ICLs as part of the Fanconi Anemia pathway.

\section{ROLE OF SUMO-LIKE DOMAINS (SLDS) IN DUB-SUBSTRATE INTERACTIONS}

While the importance of USP1/UAF1-mediated deubiquitylation of FANCD2 has been clearly established (Nijman et al., 2005; Oestergaard et al., 2007; Kim et al., 2009), the question of how USP1/UAF1 is targeted to the FANCI/FANCD2 heterodimer has remained elusive until recently. Yang et al. (2011) discovered that this targeting mechanism involves SUMOlike domains (SLD1 and SLD2) at the C-terminus of UAF1, which bind directly to SIM motifs of FANCI. Likewise, the SLD2 domain of UAF1 also binds to the SIM on hELG1 to direct USP1/UAF1 binding to another important substrate, PCNA-Ub (Huang et al., 2006). This SLD-SIM interaction is critical for FA pathway function, as deletion of the SLD2 sequence of UAF1 or the SIM of FANCI leads to deficient FANCD2 monoubiquitylation and DNA repair. Thus, SLDSIM interactions provide a means for the regulated delivery of USP1/UAF1 DUB complex to its substrates for efficient ICL repair (Yang et al., 2011) (Figure 2E). As UAF1 is a highly abundant protein with many diverse binding partners, this study also points to the possibility that SLD-SIM targeting may also play broader roles not only in DNA repair, but as a general means to sort intracellular proteins involved in other processes.

\section{FUTURE PERSPECTIVES}

In summary, the above studies clearly demonstrate the importance of SUMO and SUMO-like modifications in finetuning FA pathway activation and DNA repair (summarized in Figure 2). It is probable that findings reported here represent a small portion of targets controlled by SUMOylation in the FA pathway, and future work should uncover other unidentified substrates and SUMO ligases/proteases critical to this process. These studies also point to the possibility of pharmacologically targeting SUMO ligases, SIM-SUMO interactions, and SENPs as a way to manipulate FA pathway activity in FA cells and HR-defective cancers. Consistent with this notion, several studies have reported the overexpression of SENPs in various disease conditions and cancers, prompting recent advancements in the development of small molecule inhibitors of SENPs with therapeutic potential (review in Kumar and Zhang, 2015). On the other hand, aggressive modulation of SUMO- 
ubiquitin signaling could also pose a risk for inefficient repair by the FA pathway and other DNA repair mechanisms. Thus, future studies are highly necessary to further understand the proper balance of SUMOylation and ubiquitination activity necessary for proper FA pathway function, and how dysregulation of these ubiquitin and ubiquitin-like post-translational modifiers underlies genome instability.

\section{AUTHOR CONTRIBUTIONS}

KC wrote the manuscript with advice from $\mathrm{TH}$.

\section{REFERENCES}

Andersen, S. L., Bergstralh, D. T., Kohl, K. P., LaRocque, J. R., Moore, C. B., and Sekelsky, J. (2009). Drosophila MUS312 and the vertebrate ortholog BTBD12 interact with DNA structure-specific endonucleases in DNA repair and recombination. Mol. Cell 35, 128-135. doi: 10.1016/j.molcel.2009.06.019

Budzowska, M., Graham, T. G., Sobeck, A., Waga, S., and Walter, J. C. (2015). Regulation of the Rev1-pol $\zeta$ complex during bypass of a DNA interstrand cross-link. EMBO J. 34, 1971-1985. doi: 10.15252/embj.201490878

Ciccia, A., Ling, C., Coulthard, R., Yan, Z., Xue, Y., Meetei, A. R., et al. (2007). Identification of FAAP24, a Fanconi anemia core complex protein that interacts with FANCM. Mol. Cell 25, 331-343. doi: 10.1016/j.molcel.2007.01.003

Cohn, M. A., Kowal, P., Yang, K., Haas, W., Huang, T. T., Gygi, S. P., et al. (2007). A UAF1-containing multisubunit protein complex regulates the Fanconi anemia pathway. Mol. Cell 28, 786-797. doi: 10.1016/j.molcel.2007.09.031

Duxin, J. P., and Walter, J. C. (2015). What is the DNA repair defect underlying Fanconi anemia? Curr. Opin. Cell Biol. 37, 49-60. doi: 10.1016/j.ceb.2015.09.002

Fekairi, S., Scaglione, S., Chahwan, C., Taylor, E. R., Tissier, A., Coulon, S., et al. (2009). Human SLX4 Is a Holliday junction resolvase subunit that binds multiple DNA repair/recombination endonucleases. Cell 138, 78-89. doi: 10.1016/j.cell.2009.06.029

Galanty, Y., Belotserkovskaya, R., Coates, J., and Jackson, S. P. (2012). RNF4, a SUMO-rageted ubiquitin E3 ligase, promotes DNA double-strand break repair. Genes Dev. 26, 1179-1195. doi: 10.1101/gad.188284.112

Galanty, Y., Belotserkovskaya, R., Coates, J., Polo, S., Miller, K. M., and Jackson, S. P. (2009). Mammalian SUMO E3-ligases PIAS1 and PIAS4 promote responses to DNA double-strand breaks. Nature 462, 935-939. doi: 10.1038 /nature 08657

Garcia-Higuera, I., Taniguchi, T., Ganesan, S., Meyn, M. S., Timmers, C., Hejna, J., et al. (2001). Interaction of the Fanconi anemia proteins and BRCA1 in a common pathway. Mol. Cell 7, 249-262. doi: 10.1016/S1097-2765(01)00173-3

Gareau, J. R., and Lima, C. D. (2010). The SUMO pathway: emerging mechanisms that shape specificity, conjugation and recognition. Nat. Rev. Mol. Cell Biol. 11, 861-871. doi: 10.1038/nrm3011

Gibbs-Seymour, I., Oka, Y., Rajendra, E., Weinert, B. T., Passmore, L. A., Patel, K. J., et al. (2015). Ubiquitin-SUMO circuitry controls activated fanconi anemia ID complex dosage in response to DNA damage. Mol. Cell 57, 150-164. doi: 10.1016/j.molcel.2014.12.001

González-Prieto, R., Cuijpers, S. A. G., Luijsterburg, M. S., van Attikum, H., and Vertegaal, A. C. O. (2015). SUMOylation and PARylation cooperate to recruit and stabilize SLX4 at DNA damage sites. EMBO Rep. 16, 512-519. doi: 10.15252/embr.201440017

Guervilly, J. H., Takedachi, A., Naim, V., Scaglione, S., Chawhan, C., Lovera, Y., et al. (2015). The SLX4 complex is a SUMO E3 ligase that impacts on replication stress outcome and genome stability. Mol. Cell 57, 123-137. doi: 10.1016/j.molcel.2014.11.014

Guzzo, C. M., Berndsen, C. E., Zhu, J., Gupta, V., Datta, A., Greenberg, R. A., et al. (2012). RNF4-dependent hybrid SUMO-ubiquitin chains are signals for RAP80 and thereby mediate the recruitment of BRCA1 to sites of DNA damage. Sci. Signal. 5:ra88. doi: 10.1126/scisignal.2003485

Hanada, K., Budzowska, M., Modesti, M., Maas, A., Wyman, C., Essers, J., et al. (2006). The structure-specific endonuclease Mus81-Emel promotes conversion

\section{FUNDING}

Support was provided by the National Institutes of Health (NIH), American Cancer Society (ACS), NYU Laura and Isaac Perlmutter Cancer Center Support Grant's Developmental Project Program, and Irma T. Hirschl Career Scientist Award.

\section{ACKNOWLEDGMENT}

We also thank K. Burns-Huang and members of the Huang lab for critical reading of the manuscript.

of interstrand DNA crosslinks into double-strands breaks. EMBO J. 25, 4921-4932. doi: 10.1038/sj.emboj.7601344

$\mathrm{Hu}, \mathrm{X} .$, Paul, A., and Wangs, B. (2012). Rap80 protein recruitment to DNA double-strand breaks requires binding to both small ubiquitin-like modifier (SUMO) and ubiquitin conjugates. J. Biol. Chem. 287, 25510-25519. doi: 10.1074/jbc.M112.374116

Huang, T. T., Nijman, S. M. B., Mirchandani, K. D., Galardy, P. J., Cohn, M. A., Haas, W., et al. (2006). Regulation of monoubiquitinated PCNA by DUB autocleavage. Nat. Cell Biol. 8, 339-347. doi: 10.1038/ncb1378

Kee, Y., and D'Andrea, A. D. (2012). Molecular pathogenesis and clinical management of Fanconi anemia. J. Clin. Invest. 122, 3799-3806. doi: 10.1172/JCI58321

Kim, H., and D'Andrea, A. D. (2012). Regulation of DNA cross-link repair by the Fanconi anemia/BRCA pathway. Genes Dev. 26, 1393-1408. doi: 10.1101/gad.195248.112

Kim, H., Yang, K., Dejsuphong, D., and D'Andrea, A. D. (2012). Regulation of Rev1 by the Fanconi anemia core complex. Nat. Struct. Mol. Biol. 19, 164-170. doi: 10.1038/nsmb.2222

Kim, J. M., Kee, Y., Gurtan, A., and Andrea, A. D. D. (2008). Cell cycle - dependent chromatin loading of the Fanconi anemia core complex by FANCM / FAAP24. Blood 111, 5215-5222. doi: 10.1182/blood-2007-09-113092

Kim, J. M., Parmar, K., Huang, M., Weinstock, D. M., Ruit, C. A., Kutok, J. L., et al. (2009). Inactivation of murine Uspl results in genomic instability and a Fanconi anemia phenotype. Dev. Cell 16, 314-320. doi: 10.1016/j.devcel.2009.01.001

Klein Douwel, D., Boonen, R. A., Long, D. T., Szypowska, A. A., Räschle, M., Walter, J. C., et al. (2014). XPF-ERCC1 Acts in Unhooking DNA Interstrand Crosslinks in Cooperation with FANCD2 and FANCP/SLX4. Mol. Cell 54, 460-471. doi: 10.1016/j.molcel.2014.03.015

Knipscheer, P., Räschle, M., Smogorzewska, A., Enoiu, M., Ho, T. V., Schärer, O. D., et al. (2009). The Fanconi anemia pathway promotes replicationdependent DNA interstrand cross-link repair. Science 326, 1698-1701. doi: 10.1126/science. 1182372

Kumar, A., and Zhang, K. Y. J. (2015). Advances in the development of SUMO specific protease (SENP) inhibitors. Comput. Struct. Biotechnol. J. 13, 204-211. doi: 10.1016/j.csbj.2015.03.001

Leung, J. W. C., Wang, Y., Fong, K. W., Huen, M. S. Y., Li, L., and Chen, J. (2012). Fanconi anemia (FA) binding protein FAAP20 stabilizes FA complementation group A (FANCA) and participates in interstrand cross-link repair. Proc. Natl. Acad. Sci. U.S.A. 109, 4491-4496. doi: 10.1073/pnas.1118720109

Ling, C., Ishiai, M., Ali, A. M., Medhurst, A. L., Neveling, K., Kalb, R., et al. (2007). FAAP100 is essential for activation of the Fanconi anemiaassociated DNA damage response pathway. EMBO J. 26, 2104-2114. doi: 10.1038/sj.emboj.7601666

Martin, R. W., Orelli, B. J., Yamazoe, M., Minn, A. J., Takeda, S., and Bishop, D. K. (2007). RAD51 up-regulation bypasses BRCA1 function and is a common feature of BRCA1-deficient breast tumors. Cancer Res. 67, 9658-9665. doi: 10.1158/0008-5472.CAN-07-0290

Meetei, A. R., de Winter, J. P., Medhurst, A. L., Wallisch, M., Waisfisz, Q., van de Vrugt, H. J., et al. (2003). A novel ubiquitin ligase is deficient in Fanconi anemia. Nat. Genet. 35, 165-170. doi: 10.1038/ng1241

Moldovan, G. L., Dejsuphong, D., Petalcorin, M. I. R., Hofmann, K., Takeda, S., Boulton, S. J., et al. (2012). Inhibition of homologous recombination 
by the PCNA-interacting protein PARI. Mol. Cell 45, 75-86. doi: 10.1016/j.molcel.2011.11.010

Morris, J. R., Boutell, C., Keppler, M., Densham, R., Weekes, D., Alamshah, A., et al. (2009). The SUMO modification pathway is involved in the BRCA1 response to genotoxic stress. Nature 462, 886-890. doi: 10.1038/nature08593

Moynahan, M. E., and Jasin, M. (2010). Mitotic homologous recombination maintains genomic stability and suppresses tumorigenesis. Nat. Rev. Mol. Cell Biol. 11, 196-207. doi: 10.1038/nrm2851

Munoz, I. M., Hain, K., Declais, A. C., Gardiner, M., Toh, G. W., SanchezPulido, L., et al. (2009). Coordination of structure-specific nucleases by human SLX4/BTBD12 is required for DNA repair. Mol. Cell 35, 116-127. doi: 10.1016/j.molcel.2009.06.020

Nakanishi, K., Yang, Y., Pierce, A. J., Taniguchi, T., Digweed, M., Andrea, A. D. D., et al. (2005). Human Fanconi anemia monoubiquitination pathway promotes homologous DNA repair. Proc. Natl. Acad. Sci. U.S.A. 102, 1110-1115. doi: 10.1073/pnas.0407796102

Nijman, S. M. B., Huang, T. T., Dirac, A. M. G., Brummelkamp, T. R., Kerkhoven, R. M., D’Andrea, A. D., et al. (2005). The deubiquitinating enzyme USP1 regulates the Fanconi anemia pathway. Mol. Cell 17, 331-339. doi: 10.1016/j.molcel.2005.01.008

Noll, D. M., Mason, T. M., and Miller, P. S. (2006). Formation and repair of interstrand cross-links in DNA. Chem. Rev. 106, 277-301. doi: $10.1021 / \mathrm{cr} 040478 \mathrm{~b}$

Oestergaard, V. H., Langevin, F., Kuiken, H. J., Pace, P., Niedzwiedz, W., Simpson, L. J., et al. (2007). Deubiquitination of FANCD2 is required for DNA crosslink repair. Mol. Cell 28, 798-809. doi: 10.1016/j.molcel.2007.09.020

Ouyang, J., Garner, E., Hallet, A., Nguyen, H. D., Rickman, K. A., Gill, G., et al. (2015). Noncovalent interactions with SUMO and ubiquitin orchestrate distinct functions of the SLX4 complex in genome maintenance. Mol. Cell 57, 108-122. doi: 10.1016/j.molcel.2014.11.015

Papouli, E., Chen, S., Davies, A. A., Huttner, D., Krejci, L., Sung, P., et al. (2005). Crosstalk between SUMO and ubiquitin on PCNA is mediated by recruitment of the helicase Srs2p. Mol. Cell 19, 123-133. doi: 10.1016/j.molcel.2005.06.001

Perry, J. J. P., Tainer, J. A., and Boddy, M. N. (2008). A SIM-ultaneous role for SUMO and ubiquitin. Trends Biochem. Sci. 33, 201-208. doi: 10.1016/j.tibs.2008.02.001

Qiao, F., Moss, A., and Kupfer, G. M. (2001). Fanconi anemia proteins localize to chromatin and the nuclear matrix in a DNA damage- and Cell cycle-regulated manner. J. Biol. Chem. 276, 23391-23396. doi: 10.1074/jbc.M101855200

Räschle, M., Knipscheer, P., Knipsheer, P., Enoiu, M., Angelov, T., Sun, J., et al. (2008). Mechanism of replication-coupled DNA interstrand crosslink repair. Cell 134, 969-980. doi: 10.1016/j.cell.2008.08.030

Rodriguez, M. S., Dargemont, C., and Hay, R. T. (2001). SUMO-1 conjugation in vivo requires both a consensus modification motif and nuclear targeting. J. Biol. Chem. 276, 12654-12659. doi: 10.1074/jbc.M009476200
Sims, A. E., Spiteri, E., Sims, R. J., Arita, A. G., Lach, F. P., Landers, T., et al. (2007). FANCI is a second monoubiquitinated member of the Fanconi anemia pathway. Nat. Struct. Mol. Biol. 14, 564-567. doi: 10.1038/ nsmb1252

Singh, T. R., Saro, D., Ali, A. M., Zheng, X. F., Du, C. H., Killen, M. W., et al. (2010). MHF1-MHF2, a histone-fold-containing protein complex, participates in the Fanconi anemia pathway via FANCM. Mol. Cell 37, 879-886. doi: 10.1016/j.molcel.2010.01.036

Smogorzewska, A., Matsuoka, S., Vinciguerra, P., McDonald, E. R., Hurov, K. E., Luo, J., et al. (2007). Identification of the FANCI protein, a monoubiquitinated FANCD2 paralog required for DNA repair. Cell 129, 289-301. doi: 10.1016/j.cell.2007.03.009

Stoepker, C., Hain, K., Schuster, B., Hilhorst-Hofstee, Y., Rooimans, M. A., Steltenpool, J., et al. (2011). SLX4, a coordinator of structure-specific endonucleases, is mutated in a new Fanconi anemia subtype. Nat. Genet. 43, 138-141. doi: 10.1038/ng.751

Walden, H., and Deans, A. J. (2014). The Fanconi anemia DNA repair pathway: structural and functional insights into a complex disorder. Annu. Rev. Biophys. 43, 257-278. doi: 10.1146/annurev-biophys-051013022737

Wan, B., Yin, J., Horvath, K., Sarkar, J., Chen, Y., Wu, J., et al. (2013). SLX4 assembles a telomere maintenance toolkit by bridging multiple endonucleases with telomeres. Cell Rep. 4, 861-869. doi: 10.1016/j.celrep.2013. 08.017

Xie, J., Kim, H., Moreau, L. A., Puhalla, S., Garber, J., Abo, M. A., et al. (2015). RNF4-mediated polyubiquitination regulates the Fanconi anemia/BRCA pathway. J. Clin. Invest. 125, 1523-1532. doi: 10.1172/JCI79325

Yan, Z., Delannoy, M., Ling, C., Daee, D., Osman, F., Muniandy, P. A., et al. (2010). A histone-fold complex and FANCM form a conserved DNAremodeling complex to maintain genome stability. Mol. Cell 37, 865-878. doi: 10.1016/j.molcel.2010.01.039

Yang, K., Moldovan, G. L., Vinciguerra, P., Murai, J., Takeda, S., and D’Andrea, A. D. (2011). Regulation of the Fanconi anemia pathway by a SUMO-like delivery network. Genes Dev. 25, 1847-1858. doi: 10.1101/gad.17020911

Conflict of Interest Statement: The authors declare that the research was conducted in the absence of any commercial or financial relationships that could be construed as a potential conflict of interest.

Copyright $\odot 2016$ Coleman and Huang. This is an open-access article distributed under the terms of the Creative Commons Attribution License (CC BY). The use, distribution or reproduction in other forums is permitted, provided the original author(s) or licensor are credited and that the original publication in this journal is cited, in accordance with accepted academic practice. No use, distribution or reproduction is permitted which does not comply with these terms. 